\title{
Le quasi-contrat du combat suicidaire
}

The quasi-contract of suicidal combat

\section{Louis-Jean DUCLOS}

\section{(2) OpenEdition}

1 Journals

\section{Édition électronique}

URL : http://journals.openedition.org/conflits/2096

DOI : 10.4000/conflits.2096

ISSN : $1777-5345$

Éditeur :

CCLS - Centre d'études sur les conflits lilberté et sécurité, L'Harmattan

\section{Édition imprimée}

Date de publication : 1 novembre 2006

Pagination : 25-46

ISBN : 2-296-01436-4

ISSN : 1157-996X

\section{Référence électronique}

Louis-Jean DUCLOS, " Le quasi-contrat du combat suicidaire », Cultures \& Conflits [En ligne], 63 | automne 2006, mis en ligne le 01 décembre 2006, consulté le 30 mars 2021. URL : http:// journals.openedition.org/conflits/2096 ; DOI : https://doi.org/10.4000/conflits.2096

Ce document a été généré automatiquement le 30 mars 2021

Creative Commons License 


\title{
Le quasi-contrat du combat suicidaire
}

The quasi-contract of suicidal combat

\author{
Louis-Jean DUCLOS
}

«Chaque sorte de suicide n'est donc que la forme exagérée ou déviée d'une

vertu ${ }^{1} »$.

«On voudrait crever puisqu'on ne peut faire crever les autres ${ }^{2}$ ".

1 Le 10 septembre 2004, à la veille d'un anniversaire fameux pour l'histoire du suicide de combat, s'est produit un micro-événement propre à illustrer sa problématique. Ce jourlà, à Cancun, au premier jour de la cinquième conférence ministérielle de l'Organisation mondiale du commerce, le militant altermondiste Lee Kyung-hae s'est donné la mort à la tête d'une manifestation paysanne de protestation. Il accomplissait ce faisant, avec toute la dramatique solennité du "martyre ${ }^{3}$ ", un geste de protestation politique ${ }^{4}$ et, plus spécifiquement, un suicide accusatoire. L'opinion dans son ensemble préféra y voir le geste d'un déséquilibré. Pour légère et sotte qu'elle soit dans son réductionnisme, cette réaction révèle un refus assez général d'envisager la complexité d'une conduite qui dérange. $Y$ voir le symptôme d'une pathologie "vésanique", comme dirait Durkheim ${ }^{5}$, est évidemment simplificateur à l'excès, dans la mesure où un tel acte, fondé en idéologie, préparé en logistique (fût-elle sommaire), ordonné à un objectif et inscrit de surcroît dans une longue tradition, peut difficilement passer pour l'expression paroxystique d'une monomanie.

2 Les suicides de combat couvrent un large spectre, allant du suicide collectif « défensif » au suicide individuel «terroriste ${ }^{6}$ ». En font également partie ces suicides polémiques aux effets mobilisateurs indirects, dont la seule victime proche est son auteur luimême. Peu de choses en commun en effet, quoique tous "politiques ", entre l'attentat terroriste non ciblé et l'auto-immolation des zélotes de Massada (70 apr. J.-C.) ou le sacrifice ${ }^{7}$ déclamatoire du député Alphonse Baudin en 1851, qui voulait montrer à ses concitoyens, comment, en tant que député républicain, «on meurt pour 25 francs par jour ${ }^{8}$ » ou encore l'harakiri de Lee Kyung-hae. Il s'agirait cependant dans tous les cas de ces suicides "altruistes obligatoires", selon la typologie durkheimienne ${ }^{9}$, vécus 
comme des devoirs, au terme desquels le sujet sacrifie à l'image qu'on lui a appris à avoir de lui-même : plutôt mourir que de survivre au " déshonneur »; sombrer avec la République quand on est républicain et/ou avec le bateau qui coule quand on en est le commandant, plutôt que de survivre à leur naufrage.

3 Ces suicides impliquent, à n'en pas douter, un fort degré de socialisation-exclusion tendant à fusionner idéalement le suicidant au groupe idéologique d'appartenance et à déshumaniser sa relation avec tout le reste, qu'il s'agisse d'endeuiller sa propre mère éplorée ou d'exterminer des innocents dans des conditions passablement odieuses. Si réprouvé que soit en l'occurrence ce modus operandi, il faut cependant le considérer d'abord d'un point de vue polémologique comme une technique, le sacrifice de soi n'étant alors que l'une des modalités du combat. Ainsi du Samson biblique, s'ensevelissant sous les ruines d'un temple de Gaza pour entraîner des Philistins dans la mort (xie siècle av. J.-C.), du jihadiste Mohammed Atta, co-auteur des attentats du 11 septembre, en passant par les kamikazes japonais de la Seconde Guerre mondiale, ou Yan Palach s'immolant par le feu pour protester contre l'entrée des troupes du Pacte de Varsovie en Tchécoslovaquie en 1968, la liste est à jamais ouverte de ces personnages « héroïques » qui, dans une perspective polémique, se servent du suicide comme d'une arme.

4 A défaut d'entrer sans préjugés dans le sujet, il importe du moins de l'aborder en se gardant des émotions et des phobies. Que le sacrifice du Christ, pour pacifiste qu'on le suppose, puisse être comparé aux « morts au champ d'honneur » expirant dans les bras de leur aumônier en invoquant le Bon Dieu et leur maman, pour ne rien dire évidemment du suicidaire terroriste, pourrait paraitre au lecteur comme le dernier blasphème. Il n'en reste pas moins qu'il faut élucider si la mort volontaire du Christ pour l'avènement sur terre du Royaume des Cieux entre dans notre problématique, et sinon, quelle spécificité l'en exclut?

5 On ne saurait donc faire l'économie d'une réflexion typologique préalable qui permette de dépasser la morphologie d'un phénomène dont l'actualité laisse deviner le potentiel apocalyptique, sans pour autant rien révéler de sa dimension éthique, sociale et politique. Bien après la classification proposée par Durkheim en 1897 en suicides " égoïstes", "altruistes", et "anomiques» et largement critiquée depuis, Jean Baechler - dont la thèse "passionnante", selon Raymond Aron fait autorité distingue, quant à lui, onze espèces de suicides relevant de quatre genres ${ }^{10}$. On aurait pu tenter aussi une approche multi-polarisée du suicide de combat selon des axes oscillants, aux extrémités desquels figureraient les contraires actif / passif; protestataire symbolique / meurtrier terroriste; individuel / collectif; offensif / défensif, etc. Pour mettre de l'ordre dans cette profusion, Maurice Pinguet, spécialiste du suicide à la japonaise, s'y est pour sa part essayé ${ }^{11}$. Nous en avons tiré le schéma suivant : 


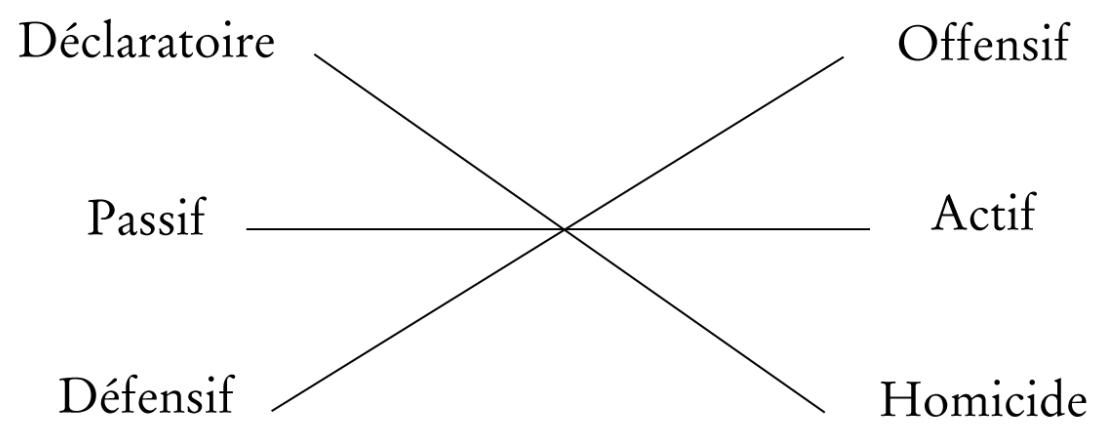

6 Tel est l'échiquier mobile sur lequel évoluent le suicidant, ses cibles, et les tiers, dans une relation stratégique incertaine.

\section{Acteurs, tiers et société}

7 Si toute violence est dramatique, le suicide polémique l'est triplement : par ses objectifs visant à bouleverser un rapport de forces, par son modus operandi à l'encontre duquel le sens commun se révolte, par les victimes parfaitement hasardeuses qu'il atteint parfois. S'il évoque quelquefois, notamment par analogie avec certaines violences de droit commun, la notion de contrat explorée plus loin, il s'inscrit nécessairement dans un climat de crise affectant acteurs, témoins et société. Il pose tout d'abord la question $\mathrm{du}$ rapport de l'agent suicidant avec une base sociale, au front de laquelle figurent tiers et témoins. Selon, en effet, que cette relation s'inscrive dans le consensus ou, au contraire, en rupture avec l'environnement idéologique dominant, elle favorisera l'émergence de comportements suicidaires héroïques de masse ou l'éclatement en groupuscules s'auto-justifiant, mûrs pour l'action terroriste. L'inhibition de l'instinct individuel de conservation est leur dénominateur commun et constitue l'archétype du suicide altruiste, moins sous l'effet d'un processus de dépersonnalisation propre, selon Durkheim, au "primitif ${ }^{12}$ ", que par l'appropriation individuelle un effet d'individualisation de causes générales.

8 Les puissantes motivations qui conduisent le suicidant au passage à l'acte couvrent un large spectre allant du culturel apparemment le plus gratuit à l'événementiel le plus bouleversant. A l'horizon du suicide de combat apparemment le plus vain, celui qui, à court terme, ne meurtrit en rien l'adversaire - se donner la mort ou se la faire donner sans se préoccuper d'en faire payer comptant le prix à quiconque - renverrait à une valeur essentielle pour le guerrier : l'honneur ${ }^{13}$. Pas seulement du guerrier, du militant politique aussi, et même de l'être social moyen. Ainsi la femme violée au détour d'une bataille se tuera pour ne pas survivre à l'infamie subie. Ainsi Caton d'Utique, en deuil de la République romaine.

9 A cet impératif culturel qui fait de la mort volontaire un devoir, se mêlent, à l'occasion, des mobiles plus circonstanciels dont François Géré a tenté l'inventaire : le dénuement économique ${ }^{14}$, un endoctrinement spécifique, le charisme d'un chef, le désir de vengeance, la dénonciation, le défi, la haine, la frustration, l'attrait d'une récompense, l'auto-exaltation révolutionnaire, la drogue, le droit même et son corollaire, le 
gendarme, etc. ${ }^{15} \mathrm{~A}$ cela s'ajoute éventuellement l'effet de groupe mettant en œuvre des réflexes d'imitation, de contagion et d'émulation qui peuvent atteindre dans une configuration militaire des dimensions sidérantes: des Thermopyles à Stalingrad, le nombre des victimes dont beaucoup eurent un comportement héroïco-suicidaire de « conformité » a atteint 300 000-350 000, sans oublier les 700000 sacrifiés de Verdun.

Il va sans dire que, dans une conjoncture aussi mortifère, l'idéologie en général et les religions en particulier jouent un rôle d'adjuvants si important qu'elles sont fortement sollicitées par les entrepreneurs et les pratiquants du suicide combattant, au prix parfois d'acrobaties casuistiques qu'il n'est pas dans notre propos d'analyser ici. Les "religions laïques ", moins encombrées de tradition sont, à cet égard, plus à l'aise. Certaines, KGB aidant, furent, comme le marxisme soviétique, extrêmement pourvoyeuses de morts volontaires, d'autres, comme le nihilisme de Stavroguine - qui prêchait à son disciple l'indifférence à la vie pour le bonheur de devenir dieu ${ }^{16}-$ le furent moins. Quant aux systèmes de croyances qui introduisent l'homme au surnaturel, ils sont d'une efficacité éprouvée, limitée en ce qui concerne les religions dites «du Livre» (christianisme, islam, judaïsme), par une révélation littérale contraignante, mais largement ouverte aux manipulations exégétiques. La propension des entrepreneurs de suicides à solliciter les religions, dans la mesure où celles-ci promettent qu'on vive après la mort, se manifeste aujourd'hui tout particulièrement dans l'aire islamique. Sur cinq conflits actuels mettant couramment en œuvre des techniques suicidaires - Israël-Palestine, Russie-Tchétchénie, Cachemire-Inde, Al-Qaïda - Occident, Tamoul-Sri Lanka -, la référence islamique est fortement sollicitée dans les quatre premiers cas. On aurait tort toutefois d'en conclure au caractère intrinsèquement suicidogène de l'islam ${ }^{17}$ car c'est l'acuité du conflit qui interpelle la religion et pousse à des interprétations transgressives, et non l'inverse.

11 Plus que des religions qui ne sont, la plupart du temps, que l'habillage sacralisant d'une pratique prosaïque mais littéralement extraordinaire, une impulsion ou un encouragement spécifique est à attendre des bases sociales constituées en vue précisément du combat. Elles résultent d'organisations allant des forces armées dûment instituées aux groupes clandestins révolutionnaires les plus marginaux, souvent les plus fanatiques. Des unes aux autres, à l'exclusion des nihilistes les plus radicaux et des régimes matérialistes qui se disent scientifiques, on croit aussi à une survie située, sinon dans des paradis peuplés ou non d'hypothétiques jeunes vierges, du moins dans la mémoire des hommes. La voie du sacrifice de soi n'en est pas moins ardue, mais le conditionnement idéologique n'emprunte pas les mêmes chemins. Le rapport au donneur d'ordre ou à l'autorité de référence, qu'il soit le chef institutionnel ou, comme on dit par manière discrètement diffamante, le " commanditaire », n'est également pas non plus le même. Aussi y a-t-il lieu de distinguer entre les deux pôles de l'engagement combattant: le "guerrier » incorporé dans une structure militaire d'Etat et le résistant-révolutionnaire membre de groupuscules clandestins à la légitimité contestée. Quelque part entre les deux se situent le mercenaire contractuel et l'activiste d'organisations semi-légitimes dénommées « terroristes » par les uns ou « libératrices » par d'autres. 


\section{L'« exception » militaire}

12 L'institution militaire est le lieu par excellence où s'enseignent, se développent et s'incarnent les notions d'honneur, de devoir et de sacrifice ${ }^{18}$, mais aussi d'obéissance, longtemps enseignée comme inconditionnelle. Simultanément cependant, le suicide y est tenu en une telle aversion que la plupart des armées en répriment les tentatives. Ce paradoxe apparent entre économie et don de soi, tient à ce que le suicide "égoïste ", par lequel le suicidant n'a en vue que des objectifs personnels, est évalué techniquement comme une perte d'effectif, et moralement comme une lâcheté. Il inspire ce principe élémentaire de l'enseignement tactique de base : « tuer et n'être pas tué ». Le culte des héros morts au combat, à base de cérémonies, de monuments et de discours commémoratifs, relève plus d'une technique de deuil que de la prescription d'une conduite. Littéralement, la mort d'un combattant dénote une sorte d'échec relevant soit d'un rapport de force défavorable, soit d'une circonstance accidentelle fâcheuse. Dans une certaine mesure, le geste du soldat tombé au "champ d'honneur " dans l'acceptation d'une mort volontaire est célébré bien qu'il soit mort et non parce qu'il l'est. Au début d'une guerre "fraîche et joyeuse », on entonne spontanément «la victoire en chantant !». Ce n'est qu'en envisageant plus tard les épreuves de la bataille qu'on chantera par manière d'incantation conjuratoire «mourir pour la Patrie, c'est le sort le plus beau ! » parce que ce n'est tout simplement pas vrai. Le « sort le plus beau », c'est d'être heureux, dans une patrie resplendissante sans doute, mais au moindre coût possible, notamment en vies humaines. Cette vérité de tous les temps s'est affichée tout crûment, avec autant d'arrogance que d'illusion, avec le slogan du « zéro mort » dans les armées de la coalition lors de la première guerre d'Irak en 1991. Les perspectives diffèrent sensiblement au point de paraître s'inverser quand on passe d'une situation offensive à la phase défensive d'une campagne.

" Mourir sur place plutôt que de reculer », comme l'ordonnait Joffre en 1914, est, à n'en point douter, le commandement d'une sorte de suicide. Néanmoins, une telle conduite face à un danger mortel pour la patrie, métaphoriquement reconstruite comme un foyer domestique peuplé d'épouses et d'enfants, n'est, en somme, que banale qui consiste à affronter le risque suprême pour le sauvetage des siens. Notons toutefois que l'injonction de "mourir sur place » revêt un caractère à la fois solennel et collectif qui ôte à l'exécutant de base une bonne part de libre arbitre. Ne s'agissant, en tout état de cause, que d'un risque, fut-il extrême, le suicide, programmé au plan collectif, ne l'est pas individuellement. Il serait abusif de penser avec Céline que cette disposition relève d'un manque d'imagination. Si, de fait, la part d'imitation et de soumission hiérarchique, voire de décervelement due à la fureur des combats est importante, celle, à l'opposé, du dévouement au chef, du sentiment de l'honneur personnel, de l'amour des siens ne l'est pas moins. D'Azincourt (1415) à la Guerre du Golfe (1991), en passant par la Première Guerre mondiale (1914-1918) et celle des Malouines (1982), le « courage », l'» abnégation », l'» esprit de sacrifice », la « discipline », la « solidarité », et l'» honneur ", sont, pour Claude Barrois, les ingrédients approximatifs du moi guerrier prêt à tuer et à être tué, fortement ressassés sinon explicités dans la relation milieu / motivation du combattant suicidaire en défensive. Dans l'ambiance du combat, telle du moins que les historiographes la reconstituent, c'est la non-prise de risque qui serait moralement suicidaire, en rejetant le combattant défaillant hors du groupe, dans l'opprobre réservée aux lâches. En deçà donc de l'injonction de mourir en masse dans 
des circonstances où le regard des autres raffermit le courage de chacun, la consigne implicite du chef militaire est de ne pas mourir, ce qui fait, finalement, que le volontariat suicidaire aux armées est un phénomène exceptionnel, qui place le suicidant au carrefour de ces conduites d'échappement ou d'oblation repérés par Baechler : échapper au déshonneur d'avoir survécu à sa mission, aux épreuves d'une captivité inhumaine, voire, par la défenestration ou le cyanure, aux tourments des tortionnaires; donner sa vie pour ou à la place ou en faveur de quelqu'un ${ }^{19}$.

Dans le paysage éthique de l'institution militaire, le cas des kamikazes japonais ${ }^{20}$ fait figure d'exception, dans la mesure où il se rattache, d'une part, au concept de l'honneur guerrier si prégnant dans la culture nationale nippone et, d'autre part, à une conception particulière de l'obéissance militaire. Il se distingue aussi, malgré les pièges d'un vocabulaire vulgarisé, des motivations attribuées aux "kamikazes» du 11 septembre. Si l'on en croit les propos fortement empathiques de Maurice Pinguet sur le sujet, « rien n'émoussait pour eux le tranchant de la mort ", ni récompense, ni paradis, ni même l'espoir d'une victoire ${ }^{21}$. La «discipline» en tant que contrainte institutionnelle laissait la place à une espèce de manipulation, de sorte qu'il aurait eu paradoxalement plus de courage à résister qu'à céder au «sentiment de solidarité et d'émulation si violente à vingt ans " organisé par le commandement. Dans leur mort même, face à "l'agonie de la nation", ces jeunes gens, "parfaitement normaux ", pense l'auteur, ne cessaient, en toute liberté, « d'y mettre leur fierté et d'y trouver, en somme, toutes leurs raisons de vivre ${ }^{22} »$. Un patriotisme incandescent, certes, mais aussi le poids d'une tradition qui ne craint pas de célébrer la mort précoce et inutile "comme [celle de] la fleur de cerisier qui n'attend pas de se faner sur l'arbre ${ }^{23}$ ", seraient ainsi, estime Pinguet, à l'origine de ce «cristal de l'abnégation ». Il est d'une pureté si « déchirante ${ }^{24}$ » qu'il a suffi à inhiber, chez tant de jeunes Japonais, toute logique de conservation individuelle.

15 Le mercenaire militaire ou militarisé est le dernier avatar de l'institution combattante régulière dont la pratique de violence politique est souvent brouillée sous l'effet d'une hâtive opprobre médiatisée. Le rapport de ces contractuels à la mort combattante volontaire n'est évidemment pas celui du soldat-citoyen-patriote qui meurt pour la patrie dans la conviction que son gouvernement est légitimement fondé à lui demander le sacrifice suprême. Qu'il soit incorporé dans une structure d'Etat par contrat direct ou qu'il soit lié à celle-ci à titre d'intérim par un entrepreneur de coercition publique ${ }^{25}$, la loyauté du mercenaire n'est due qu'à son contrat, autrement dit à la rémunération qu'il perçoit en échange de son service, et non à l'Etat-major qui l'emploie ou encore moins au pouvoir politique auquel ce dernier est subordonné. Or le dit contrat, s'il n'exclut pas le risque de mort, ne comporte certainement pas l'obligation de courir au devant d'elle, quoiqu'on puisse imaginer un mercenaire assez passionnément attaché à l'entité politique au service de laquelle il est placé pour lui consentir le don de sa vie. Réputés n'être pas des sentimentaux, on ne voit cependant pas pourquoi les mercenaires professionnalisés n'accèderaient pas à la pratique du suicide d'honneur, mentionné plus haut, ni guère davantage à ces quasi-suicides de solidarité, inspirés dans l'attaque comme dans la défense par l'attachement au chef charismatique ou par l'imitation des camarades de combat.

16 A l'ordre militaire, sous-produit fondateur d'une société bien organisée et globalement anti-suicidaire, s'oppose une société civile parfois très policée, parfois profondément désintégrée. Entre ces deux pôles, la morphologie des systèmes de violence politique 
recourant à l'arme du suicide va d'un supra-nationalisme idéologique globalisant, aux groupuscules les plus ténus et les plus sectaires, mais non les moins meurtriers. Souvent d'ailleurs ces derniers - expressions de frustrations catégorielles précises combinent, au plan des motivations, le romantisme de perspectives eschatologiques enthousiasmantes et le prosaïsme de revendications bien concrètes, faute de quoi le suicide militant, comme dans le cas du Coréen évoqué au début de cet article, demeure un acte tribunitien de témoignage à l'impact politique improbable. Il n'en va pas de même dans les mouvements civils révolutionnaires ou de libération nationale ou quasi nationale. Du fait de la répression dont ils sont l'objet et de la clandestinité à laquelle celle-ci les contraint, ces derniers ont en commun une tendance à s'encapsuler dans de micro-structures dont le délitement d'une société globale en crise favorise la prolifération. Le risque est grand de voir dégénérer certaines d'entre elles en sectes purement délirantes et/ou mafieuses. Dans un chapitre sur les mobiles qui inclinent les combattants-militants au sacrifice volontaire de leur vie, François Géré souligne la dérive "de ces petites structures qui ont recréé à leur usage une représentation substitutive du monde réel ${ }^{26} »$. A moins, après tout, qu'ils ne soient des visionnaires, ce qu'on ne saura qu'à la fin de l'Histoire, une Histoire fortement obérée, en ces temps actuels, par la réaction des acteurs et des tiers face à la qualité des cibles visées par ces suicides raisonnés.

\section{La qualification victimaire}

17 La galerie des cibles nous conduit des plus abstraites, lointaines, indirectes et symboliques à la victime de proximité la plus sanguinolente et néanmoins innocente. De leur nature dépend beaucoup la qualification du suicide qui le vise, le jugement porté sur son auteur, le modus operandi de l'acte et sa portée stratégique. Plus l'objectif visé est abstrait et général, plus les motivations et dynamiques de ceux qui cherchent à l'atteindre seront idéologiques, notamment chez ceux qui font le choix du suicide combattant. Faute aussi de cible immédiatement identifiable, de tels suicides ont un caractère d'immolation. Qualifiés par Baechler de "suicides oblatifs", une certaine émotivité populaire imprégnée de religiosité les qualifie assez improprement de " sacrifice ", dans la mesure où ce terme désigne littéralement la mise à mort plus ou moins ritualisée d'une victime en vue d'être agréable à la divinité, la crucifixion du Christ en étant, selon la tradition chrétienne, le plus auguste modèle

La nature de la cible, selon qu'elle est militaire ou civile, permet certes d'opérer une grossière distinction. Le suicide meurtrier d'un militaire visant un objectif militaire peut passer pour le comble de l'héroïsme. Néanmoins, d'autres observateurs le considèreront comme d'autant plus déviant que la victime sera censée faire partie du camp des "nôtres" et son agresseur de celui de l'exotisme ensauvagé. Tel fut largement le cas du jugement porté sur la conduite des kamikazes japonais auxquels Maurice Pinguet, faisant exception, ne ménage pourtant pas le «tribut d'admiration et de compassion qu'ils méritent ${ }^{27} \%$. Il en irait d'ailleurs de même, toute notion d'agression suicidaire mise à part, lorsque les victimes, toutes civiles qu'elles soient, relèvent du camp d'en face et que les agents de leur trépas, fût-il massif, relèvent des organisations militaires de défense des «justes causes»: ainsi le bombardement des objectifs civils allemands de la Seconde Guerre mondiale. 
19 Le meurtre par assaillant suicidaire du civil désarmé, sous la condition que la victime soit perçue comme proche et l'agresseur comme relevant du sulfureux empire des barbares, est ressenti, en revanche, comme le comble de l'abomination. L'opinion occidentale en phase avec ses médias en a fourni un exemple spectaculaire avec le formidable attentat du 11 septembre 2001 ou, à un moindre degré, ceux de Madrid (11 mars 2004) et de Londres ( 7 juillet 2005), encore que, en ces occurrences, l'émotion ne relevait pas seulement de la réprobation morale, mais aussi de l'appréhension d'une vulnérabilité partagée. Plus purement moral est, à cet égard, le sentiment de révolte qu'inspirent en général les attentats-suicides anti-israéliens palestiniens. Il en va tout autrement lorsqu'assaillants et victimes se recrutent dans un même univers réputé barbare, ou tout du moins exotique, que ce soit au Sri Lanka, en Turquie, au Maroc, ou même en Tchétchénie.

On signalera enfin que la confrontation suicidant meurtrier / victime induit dans la durée un rapport dialectique d'ordre moral qui, en quelque sorte, surdétermine les représentations d'eux-mêmes et de l'adversaire que se font les acteurs, leurs proches et, partant, la nature de l'engagement des parties combattantes et leurs analyses stratégiques, l'attitude des tiers, voire l'issue de la confrontation. Là encore, il faut distinguer entre les épisodes d'un affrontement militaire conventionnel et l'apparente anomie des conflits dits civils, révolutionnaires, résistants et leurs dérives terroristes. La moralité des premiers, en effet, même lorsqu'ils s'inscrivent dans l'hébétude des mêlées de masse, garantie par l'absolution du risque partagé, est exaltée à l'envi par le commandement militaire, par "l'arrière", et les anciens combattants du lendemain. Les victimes elles-mêmes sont immolées sans haine. Dans l'arsenal des exemples édifiants, les combattants suicidaires occupent une place de choix. Combien est problématique en revanche le suicide meurtrier du «combattant de l'ombre »! Agent d'une mort artisanale tant en ce qui concerne la sienne que celle de ses victimes, sa démarche est exposée à toutes les exploitations de l'interpellation subjective. Autant la victime militaire passe pour éligible à la mort violente, autant l'attentat visant des civils, présumés plus innocents que leurs concitoyens en uniforme, ouvre les perspectives les plus complexes, tant il renvoie les antagonistes dans des systèmes sociaux aux temporalités généralement différentes et les enferme, comme l'indique Hamit Bozarslan ${ }^{28}$, dans des régimes de subjectivité qui se répondent, sans toutefois pouvoir s'entendre. Car pour surmonter l'horreur de tuer un prochain sans défense en se tuant soi-même, il y faut un apprentissage en continu de l'inhibition des morales ordinaires, de la réification de l'autre et de soi et de l'enfermement mental dans un système où n'ont d'humaine réalité qu'un au-delà ou qu'un après-soi imaginé, dans lequel les récompenses sont suffisamment garanties et correspondent à la valeur de l'enjeu ainsi qu'au coût, pour soi et pour la cible, de l'opération-suicide.

21 Quelles que soient les contraintes institutionnelles pesant sur les candidats au suicide de combat, celles-ci seraient généralement impuissantes à entrainer leur passage à l'acte, si chaque combattant suicidaire ne reconstruisait à son usage propre et exclusif un système allégorique assez puissant pour l'emporter sur ses logiques propres de conservation individuelle et dans lequel des épreuves collectives sont vécues comme autant d'épreuves personnelles, réelles ou imaginaires puissantes. 


\section{La métaphore du contrat suicidaire}

qu'inspire ce qui reste malgré tout, quelle que soit l'énormité d'autres abominations, une transgression fondamentale, contribue à brouiller d'émotion tout effort d'analyse de ce type de conduite en ce qui concerne le suicidant lui-même et son clan, la société à laquelle appartiennent les cibles et les tiers. Elle concourt en tous les cas à noyer le suicidant et l'image de celui-ci dans un système relationnel peu commun, d'où émergent les figures de chefs au charisme extraordinaire, des commanditaires mafieux ou des donneurs d'ordre aussi peu matériels que les voix de Jeanne d'Arc. Hormis le suicide "escapiste", commis en vue d'éviter des souffrances pires que la mort, se profilent au-delà d'une apparente pulsion narcissique les produits d'une culture, l'allégorie de la patrie, la statue du Commandeur, l'eschatologie laïque des lendemains radieux, le Tout-Puissant lui-même. Il se trouve sans doute que, dans l'ivresse des batailles, l'exemple $\mathrm{du}$ chef ou des camarades combattants induisent des comportements consciemment suicidaires. C'est alors seulement qu'apparaît superflue l'intervention de médiateurs spécifiques, tels qu'en requiert au contraire la prédication $\mathrm{du}$ « monde meilleur », promis par les idéologues ou le paradis des dieux décrits par les clercs.

En dépit des tendances à la sécularisation des mentalités, ces perspectives convoquent fortement l'au-delà puisque la mort du suicidant n'épuise pas son projet mais, au contraire, en conditionne l'accomplissement. Il fallait donc bien s'attendre à ce que le fait religieux fasse irruption dans cet espace articulé sur la mort, sur les promesses du salut, ainsi que sur les ascèses qui y conduisent. Le vocabulaire religieux tend alors à submerger le débat, tant par légèreté médiatique que par tendance à dérationaliser le suicidant, notamment pour le sanctifier (le "martyr») ou le diaboliser (le «terroriste »), non sans abus sémantiques plus ou moins flagrants. Ainsi banalise-t-on et gauchit-on dans le camp du «martyr » la notion de «sacrifice », pour en évacuer la dimension propitiatoire-expiatoire, traditionnellement illustrée par l'immolation de l'agneau et le mystère christique de la rédemption, dans une perspective de soumission, d'apaisement et de réconciliation. Tout à l'opposé de ce "sacrifice", le suicide à contenu polémique ne laisse plus subsister par rapport au sacré qu'une idée de rémunération : celle qui vient récompenser les «Justes » dès ce bas monde, et se parfait dans les béatitudes éternelles. Cette dérive d'un vocabulaire référé au religieux, tout en l'appauvrissant, le vulgarise, en ce qu'il tend à faire du suicide combattant le moyen d'un marché dans lequel les logiques du religieux s'inscrivent dans les contingences des psychologies, de la société et de la politique.

Ainsi posée en termes de marché, articulant coût et récompenses, la problématique de l'acte suicidaire de combat évoque l'économie d'un contrat où des parties s'obligent symétriquement, l'une " à donner, à faire ou à ne pas faire ${ }^{29}$ ", l'autre à lui en verser le prix convenu. Mais s'agit-il d'un paradigme opératoire ou d'une simple métaphore ? Or si les juristes enseignent que les trois éléments constitutifs d'un contrat bilatéral sont l'accord des contractants, l'objet du contrat et la cause de celui-ci ${ }^{30}$, ceci implique, pour que ce modèle contractuel bilatéral commutatif du donnant-donnant s'applique ici, qu'on ait affaire à des contractants, personnes (physiques ou morales) parfaitement identifiées, capables, et consentantes, s'accordant sur un objet certain, en vertu d'une cause licite. Force est de reconnaître que, si la discussion du bien-fondé de cette 
approche n'est pas dépourvue d'une certaine valeur heuristique, le paradigme contractuel ne saurait s'appliquer que de manière approximative, voire figurée.

Le schéma ci-dessous vise à proposer une interprétation graphique de ce que pourrait être un contrat suicidaire :

\section{Les co-contractants}

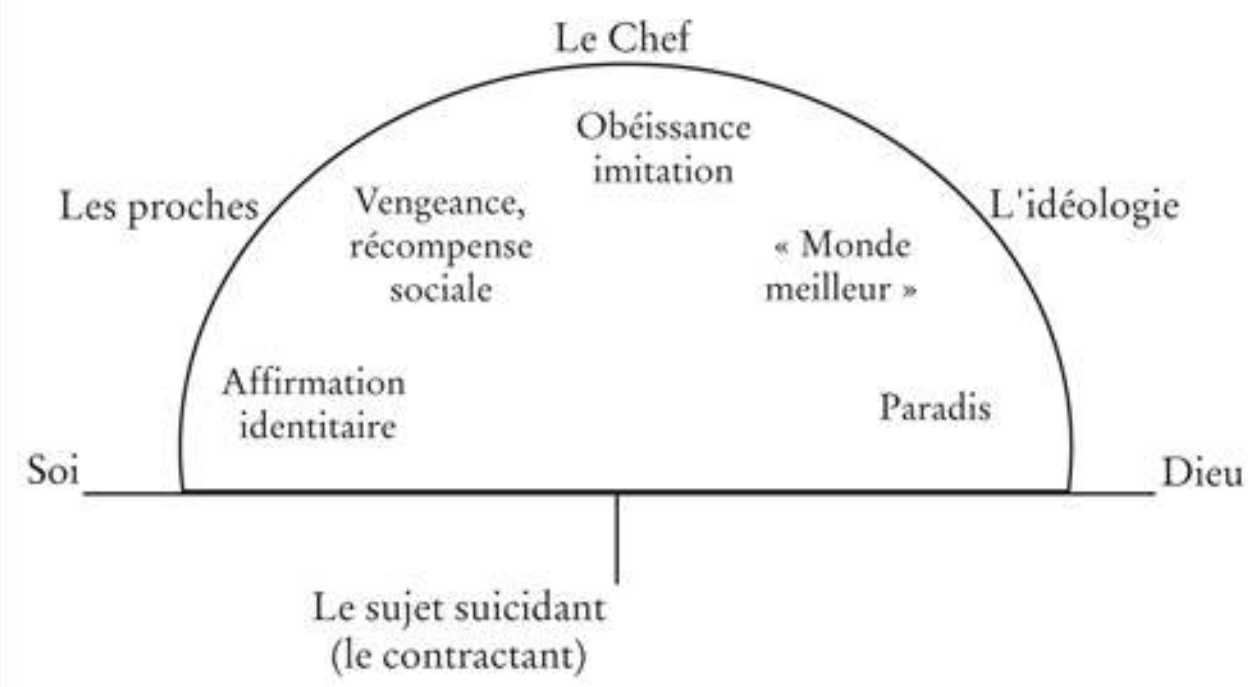

Sur ce schéma figurent, au dessus du sujet suicidant, souscripteur du supposé contrat, ses éventuels co-contractants disposés en demi-cercle allant du plus intime (soi-même) à gauche, au plus lointain, à droite (Dieu lui-même), en passant par la communauté sociale, le groupe combattant, la société politique dont relève le suicidant. Nous avons également fait apparaître, entre le suicidant et ses supposés partenaires, les principales motivations du premier, qui figureraient ainsi la cause du contrat. Il apparaît de cette manière que la relation établie entre les co-contractants progresse de l'auto-injonction narcissique d'affirmation de soi à l'aspiration la plus mystique des joies du Paradis, que les observateurs, selon leurs propres convictions, considéreront comme une production subjective ou comme une révélation surnaturelle. Entre les deux s'intercalent, sans d'ailleurs s'exclure le désir de vengeance, la considération des siens, la pression des institutions, l'autorité des chefs ou le sentiment du devoir de contribution à l'accouchement de mondes meilleurs. Il ressort d'une telle configuration que la seule certitude que nous ayons a trait à l'identité et à la réalité du sujet suicidant. La question se pose alors, en droit des contrats, de la capacité de celui-ci à souscrire un tel contrat et de la validité de son consentement.

\section{Le consentement du suicidant}

Est-il totalement et toujours sain d'esprit celui qui choisit de ne plus être pour être plus, ou s'exalte ou déprime au point de faire le choix du sacrifice certain plutôt que de la mort risquée ? La fascination du héros suicidaire ne pourrait-elle suffire à entraîner certains esprits faibles dans les voies du mimétisme? Peut-on impunément, sans risque 
de délire, demeurer à l'écoute du poète qui décrit ces sacrifiés de l'absolu, si proches et déjà si abstraits, comme des « astres scintillants, embrassant la vie sur la bouche » et qui enjoint à son compatriote : « regarde-les au loin enlacer la mort pour exister encore 31 »? Faute Faute de diagnostic fiable fondé sur l'expertise psychiatrique, force est de reconnaître que l'autonomie de la volonté du suicidant est, pour le moins, fortement exposée aux sollicitations de son histoire et aux influences de son milieu, sinon au dérèglement de ses humeurs.

Il est remarquable cependant que, du coté des suicidants comme de celui des cibles visées, les sociétés concernées répugnent à déresponsabiliser ces combattants de la mort tant, pour les besoins respectifs de leur combat, les uns ont besoin de « héros », et leurs antagonistes, d'adversaires incriminables. Jusqu'à preuve du contraire, quelle que soit l'horreur qu'elle inspire, il n'est pas d'usage de considérer la mort volontaire pour l'image de soi, pour l'honneur du groupe social, par éthique combattante, par engagement politique pour l'avènement de mondes meilleurs ou même par attrait du Paradis, comme de pure déraison.

Selon la métaphore du contrat, le consentement du contractant doit aussi, pour paraphraser notre code civil, n'être pas extorqué par la violence, c'est-à-dire par l'exercice d'une contrainte physique ou morale personnelle. On voit d'emblée les limites d'une violence physique dont l'extrême serait justement cette mort à laquelle un suicidant hésitant serait tenté de se soustraire. Laissons aussi de côté, faute d'intention polémique, ce type d'escroquerie qui prend, dans certaines sectes, l'aspect d'un conditionnement psychique proche de l'hypnose. Reste le vaste champ des représailles qu'un prescripteur ferait peser sur les proches d'un suicidant potentiel. Sans être totalement à exclure, la mise en œuvre et la portée de tels chantages ne sauraient être considérées qu'exceptionnelles.

Le consentement des contractants, disent encore les juristes, ne doit pas non plus être vicié par le dol, défini comme une tromperie sur la cause et sur l'objet du contrat, ce qui renvoie alors, sauf à de fallacieuses promesses de récompenses, à la crédulité ou à la foi du suicidant dans d'hypothétiques lendemains qui chantent « sur la terre comme au ciel », d'où un questionnement nécessaire sur l'identité du co-contractant et sa capacité à délivrer la récompense post-mortem promise. Dans le champ politique dans lequel s'inscrit «le contrat suicidaire", celle-ci, en tout état de cause, se situe bien au-delà d'une très prosaïque assurance-décès payable en gratification pécuniaire ou symbolique au profit d'un bénéficiaire désigné. Sans être totalement à exclure, il ne saurait s'agir là que de causes accessoires. L'essentiel est à chercher dans l'intériorisation d'un corpus culturel de valeurs, une allégorie politique, un au-delà surnaturel. Ainsi le co-contractant s'induit-il de la prestation attendue.

\section{Construction et promesses du co-contractant}

31 Qu'il s'agisse de l'allégorie du «champ d'honneur», du triomphe d'un projet idéologico-historique ou de la réalisation d'un plan divin, dans lequel tout croyant s'attend à tirer un jour un bénéfice ${ }^{32}$, toutes ces entités ont en commun de ne se laisser approcher par le suicidant que par des médiateurs.

Les plus ordinaires d'entre eux sont aussi les plus identifiables. Ils se comportent en fondés-de-pouvoir d'un pouvoir politique d'Etat ou d'opposition, légal ou non, engagé 
dans un combat. Ce sont eux qui promettent aux suicidants des distinctions sociales posthumes et s'engagent, souvent avec des précédents à l'appui, à attribuer à leurs survivants pensions, indemnités et honneurs publics. Parfois clandestins, ils agissent en délégués d'organisations politiques bien identifiés. Références à l'appui, ils se portent garants de l'engagement de celles-ci à délivrer, le moment venu, la récompense. $\mathrm{Ne}$ promettant toutefois que des biens matériels ou symboliques du monde présent que le suicidant s'apprête à quitter, nous ne croyons pas que leur intervention soit pour lui l'élément le plus déterminant. Il y manque à l'évidence l'élément émotionnel, seul susceptible, croyons-nous, de conduire l'individu à l'anéantissement, par amour des autres et de soi-même.

Le suicide altruiste ne se conçoit pas, en effet, sans passion dont l'objet peut être le leader charismatique ${ }^{33}$ plus ou moins relayé par ses agents ou, de manière plus sophistiquée, la cité politique en devenir, gérée et annoncée par ses idéologues et ses commissaires ou encore le "royaume des cieux", annoncé par ses clercs et ses pontifes. D'un côté, nous trouvons des personnages réels mais mythifiés comme Napoléon ou Hitler, sinon Ben Laden, qui méritent bien aux yeux des fanatisés qu'on meure pour eux; de l'autre nous avons des abstractions, des allégories, des constructions imaginaires, que les croyants disent révélées qui, elles aussi, justifient le don de soi pour la rédemption de la société réelle ${ }^{34}$, en contribution à l'avènement de la société parfaite "sur la terre comme au ciel ». Nos pinacothèques et nos cimetières regorgent de représentations des paradis s'ouvrant à nos chers défunts. Les représentations de l'immortalité laïque, de la République qui appelle au sacrifice, de la Liberté censée guider nos pas, de la mère-patrie éplorée mais exigeant néanmoins qu'on meure pour elle, ornent surabondamment nos monuments et nos espaces publics. En deçà du concept, la phonétique même du mot qui le désigne est mobilisatrice et met en branle foules et individus. Des tribuns sans scrupules en abusent. Des civilisations du Verbe s'y abîment.

En fait, face au suicidant, leaders mythifiés ou systèmes idéologiques construits n'ont guère de réalité concrète et la récompense qu'ils sont censés délivrer est compromise à plusieurs égards. Les lendemains meilleurs, les béatitudes éternelles, les jardins paradisiaques, relèvent de la croyance. Le leader mythifié, les ministres de l'utopie providentielle, les pontifes et leurs vicaires ne sont peut-être que des mystificateurs. Les promesses de récompense ne seraient alors que des leurres, auquel cas, pour en revenir à la métaphore juridique, il y aurait dol sur la cause du contrat. Pire encore, si le co-contractant du suicidant n'existe que dans son imagination, le suicidant n'a contracté qu'avec lui-même...

\section{L'objet possible, déterminé et licite du contrat}

Il semble dès lors douteux que l'objet du contrat, c'est-à-dire la matière de l'engagement des parties ${ }^{35}$, réponde aux conditions de validité des contrats retenues par la doctrine : être "possible, déterminé et licite ${ }^{36}$ ». Du coté du co-contractant rémunérateur, pour autant qu'il soit clairement identifié, des incertitudes pèsent lourdement sur sa solvabilité, sur la faisabilité et la moralité du projet politique auquel le suicidant tend à s'associer, ainsi que sur la réalité des récompenses célestes promises. Les perspectives eschatologiques ne débouchent jamais que sur de l'hypothétique, bien au-delà des certitudes fondées en raison. 

qui fait débat. Celui-ci ne porte pas sur le fait avéré qu'il aille, par hypothèse, volontairement à la mort subie ou auto-administrée, mais, en premier lieu, sur la licéité de son acte. L'acte suicidaire, en soi, fait en effet l'objet d'une réprobation morale assez générale, tout du moins dans les cultures qu'ont formées et qu'expriment les religions « monothéistes » judéo-islamo-chrétiennes. On comprend cependant que, sur le sujet des "suicides pour convenance personnelle", les législateurs séculiers soient restés sobres, faute de pouvoir envisager de répression adéquate à l'encontre d'un contrevenant déjà décédé ${ }^{37}$, sinon à l'encontre des incitateurs. trouvaient leur intérêt, la pratique de ces suicides qu'on pourrait qualifier de martiaux a souvent été placée, à l'égard des religions, dans un rapport de connivence, sinon de prescription. Ces religions ne sont-elles pas les seuls corpus idéologiques promettant aux humains une survie individuelle, paradisiaque pour les «Justes », au premier rang desquels les «martyrs »? Le vocabulaire lui-même ne contribue pas peu à entretenir la confusion. Ainsi, parler de "sacrifice» pour désigner le don de soi, spontané ou sollicité, voire imposé, du combattant, incline à une lecture religieuse et largement manipulée de phénomènes sociaux et politiques dans lesquels le rapport à Dieu est instrumentalisé. Alors que dans son acception historique l'offrande sacrificielle ne devrait avoir dans l'esprit des clercs et des fidèles qu'une portée propitiatoire généreuse en perspectives de rémunération, les pouvoirs politiques ont tôt fait de réaliser le parti qu'il pouvait tirer de ces croyances. On notera à cet égard que, des trois religions "monothéistes» - judaïsme, christianisme et islam - c'est encore de ce dernier qu'émane la condamnation doctrinale la plus catégorique qui énonce: "ne vous exposez pas à votre perte de vos mains [2/185] ${ }^{38}$ " et semble a priori la plus éloignée d'une tradition suicidaire héroïque héritée de l'antiquité gréco-latine et qu'illustre « La Garde meurt et ne se rend pas ${ }^{39}$ » du général Cambronne, décédé il est vrai en 1842 dans son lit, 27 ans après ce martial énoncé. Il n'est donc pas surprenant qu'en ce domaine et quelles que soient les religions, des entrepreneurs de violence aient toujours trouvé des aumôniers, professionnels du salut, pour justifier le sacrifice de soi sur l'autel des "nobles causes" théologiques, patriotiques, humanitaires et politiques. Le discours religieux dont s'entoure le suicide de combat facilite alors une démarche qui présente pour le sujet lui-même des aspects révoltants. C'est dire que le suicide de combat trouve plus sa cause dans la situation politique que dans le spirituel, sauf exceptions ${ }^{40}$.

Parmi celles-ci figure le mystère chrétien de la rédemption: le Christ, victime expiatoire volontaire pour le rachat des péchés du monde passant contrat avec Dieu le père lui-même, dont il était proche, pour le salut des hommes qu'il aime. Ainsi peuvent se reconnaître en cet auguste modèle ceux qui font leur en politique le principe apostolique du don de sa vie la preuve suprême d'amour pour ses frères ${ }^{41}$. Pour autant qu'un engagement politique soit aussi une forme d'amour de son prochain et qu'ici il prenne la forme d'une immolation « pacifique » - par le fer, le feu, le poison ou la grève de la faim - celle-ci, censée devoir contribuer à l'avènement d'un monde meilleur, s'interprètera comme une prise à témoin déclamatoire d'un public a priori peu mobilisé. Réduite à la dimension d'un fait divers pour la plupart des gens, la question de la légitimité de cette conduite, qui n'est exemplaire que pour quelques uns, ne se pose guère en termes de moralité civile sinon religieuse. L'immolation de soi donnée en 
spectacle ne relève-t-elle pas, après tout, d'une liberté d'expression d'autant moins contestable que son exercice réussi échappe à toute sanction? Bien plus, on crédite d'un certain panache l'auto-immolation des zélotes de Massada (70 apr. J-C.) ou les trépas du député Baudin qui préfère se faire tuer sur sa barricade plutôt que de s'en faire un abri, ou de Yan Palach (mort en 1969), arrosé d'essence ou encore Lee Kyunghae, évoqué en début d'article, du " pacha » de la corvette en perdition, qui choisit de sombrer avec son navire à moins que, comme dans le cas japonais, il ne s'agisse de suicides de remontrance conformes à la tradition confucéenne, ou de suicides de «bonnes manières » dérivés du seppuku ${ }^{42}$.

Le débat sur la licéité du suicide polémique change de nature quand ce dernier se fait meurtrier et quand sa centralité se déplace de l'acteur suicidant vers ses victimes. Encore convient-il de distinguer entre suicide de défense et suicide d'agression. Le premier n'est, au fond, qu'une des modalités du refus de déposer les armes, de se rendre à l'adversaire, d'accepter a priori le sort qu'il vous réserve, notamment la honte de la captivité. Il peut résulter explicitement de l'exécution de commandements émanant d'autorités promptes à vanter les attraits du "martyre " politique ou à affirmer, répression à l'appui, l'incontestabilité des ordres. Tuer en se tuant, dans ces conditions, passe pour d'autant plus légitime qu'une confusion absurde s'installe entre le mécanisme de la légitime défense et le suicide meurtrier en défense puisque mourir soi-même fait partie du contrat. En fait, la légitimation de ce suicide meurtrier en défense tient à ce que l'agresseur qui en est la cible est supposé recevoir ce qu'il mérite puisque, après tout, c'est lui l'agresseur. Elle s'inscrit en outre dans la culture de l'honneur militaire ou de l'abnégation militante, dans la noble tradition du contrat sacrificiel passé avec les allégories de la patrie ou d'un ordre politique meilleur. On ne sera donc pas surpris que, au sein des institutions de combat et dans les milieux qui les ont produites, ce type de suicide meurtrier en défense sans effet stratégique pour l'honneur pourrait-on dire, passe pour une norme. Le Japon en a fait une illustration surabondante dès ses premiers revers de 1943, en banalisant la conduite de ces militaires qui concluaient un combat malheureux par une charge suicidaire. Il est jusque dans nos écoles militaires les plus distinguées où on ne proposait naguère à l'imitation des futurs officiers un héros juvénile, sous-lieutenant Pol Lapeyre (décédé en 1925), qui fit « sauter son poste plutôt que de se rendre, ensevelissant à la fois sous les ruines le reste de sa garnison et ses assaillants ${ }^{43}$ ”.

$\mathrm{Si}$, dans les lignes qui précèdent, l'uniforme du capitaine de corvette et celui du souslieutenant n'introduisaient pas un élément de réflexion déterminant, c'est que les victimes de proximité intégrées à l'objet du contrat suicidaire étaient inexistantes ou disqualifiées par leur posture d'agresseurs. Il n'en va pas de même lorsque l'opérationsuicide meurtrière relève de l'initiative du suicidant, quel que soit l'état - civil ou militaire - de ses victimes. L'état militaire vaut, dans l'exercice de la violence, sinon la détention d'un monopole légal, tout au moins une présomption de légitimité. Certes, le soldat en campagne est souvent décrit comme un soudard prompt au crime dit « de guerre ", mais le danger auquel il est exposé, le cas échéant, lui vaut dans ces circonstances-là une immunité particulière et jusqu'à une certaine révérence. Selon une éthique encore vivace quoique déclinante "mourir pour la Patrie», comme le chantent les jeunes recrues, «est le sort le plus beau le plus digne d'envie » et ce en dépit des progrès des doctrines du "zéro-mort (chez-soi)» et du pacifisme postmoderne de sorte que, dans l'offensive comme en défensive, la mort au «champ d'honneur » survenant entre professionnels passe pour une chose émouvante, certes, 
mais normale. Peu importerait, dans cette perspective, que celui par qui la mort arrive se suicide ou non, dans la mesure du moins où le procédé suicidaire employé n'est pas ressenti comme relevant d'une déloyauté radicale ou d'un exotisme sauvage. A cet égard, il faut reconnaître que les kamikazes japonais (25 octobre 1944-avril 1945), vecteurs d'un « typhon divin » sont plutôt sévèrement traités.

\section{Déligitimation par la victime}

Oui, mais qu'advient-il de la licéité du contrat suicidaire quant les victimes sont des civils? Hypothèse improbable s'il s'agit d'imaginer une autorité militaire donnant l'ordre à ses soldats d'aller se tuer pour assassiner des civils « ennemis ». Elle est, au contraire fréquente, dans le cas de suicidants et de prescripteurs hors statut tel que défini par les conventions internationales relatif au droit de recours à la violence politique. En réalité, c'est le seul cas où la licéité de l'objet de notre supposé contrat suicide + mort d'autrui - se pose, bien que tranché catégoriquement par la négative sous la qualification de "terrorisme» par l'opinion dominante de la "communauté internationale». Les choses, pourtant, ne sont pas si simples: l'agent suicidant est peut-être membre d'organisations pré-étatiques qui accèderont ultérieurement à la légalité, si celles-ci sortent vainqueurs de leur combat, tels les clandestins du Yishouv pré-israélien ou les maquisards du FLN algérien. Jusqu'à ce que «l'Histoire » tranche éventuellement en sens inverse, c'est à la catégorie des «terroristes » désignée comme tels pour les tenants de l'ordre contesté qu'appartiennent les auteurs des attentatssuicides visant des civils. Les réactions émotionnelles d'horreur, de dégoût, et de haine qu'ils suscitent, non seulement dans l'environnement national des victimes mais aussi à l'horizon plus large du concert des nations dites « civilisées », débouchent sur un rejet absolu. Il est particulièrement virulent lorsque les attentats-suicides atteignent des masses d'innocents civils considérés comme proches, à New York (11 septembre 2001), Madrid (11 mars 2004) ou à Londres (7 juillet 2005). Sur un mode moins passionné, la même condamnation s'étend, par principe, aux opérations suicides confinées dans des espaces perçus comme exotiques, en Tchétchénie, au Sri Lanka, en Irak, etc.

Ce questionnement sur la moralité de l'opération suicidaire en cache en réalité un autre: celui de son utilité stratégique. Il n'échappe en effet à personne que si ses organisateurs finissaient un jour par imposer sur une aire territoriale donnée la réalisation d'un «ordre nouveau » légitimé a posteriori par la «communauté internationale", au nom de la prescription des faits anciens, le souvenir des souffrances qu'ils auraient imposées ne pèserait pas plus lourd sur la longue durée que celui des malheureuses victimes des bombardements terrifiants de Dresde ou de Nagasaki. On ne saurait non plus faire raisonnablement grief à l'appareil suicidaire qualifié aujourd'hui de "terroriste», inspirateur, commanditaires, exécutants et supporters confondus, de tuer pour des objectifs utopiques, donc en vain, car, après tout, on ne sait qu'un objectif est utopique qu'à la fin de l'histoire et ce qui passe aujourd'hui pour un crime abominable figurera demain à l'inventaire des dommages collatéraux et des œufs cassés qui ont servi à faire l'omelette. 


\section{Retour au droit positif}

Mieux vaut, en vérité, s'en tenir aux définitions du droit positif en matière de criminalité de guerre, quelles que soient par ailleurs les réticences des juristes, à l'inverse de maints politiques, à confondre guerre et terrorisme. Appliqué à ce dernier, le jus in bello offre pourtant un large éventail répressif portant notamment sur l'interdiction de la «perfidie", sur la protection des civils et de leurs biens ${ }^{44}$. Cependant, dans la pratique et contrairement aux apparences, force est de reconnaître que l'éventuelle qualification criminelle de ce type de violence, assortie des poursuites que celle-ci entraîne, tient moins au caractère civil des cibles touchées qu'à la qualité de leurs auteurs, suicidaires ou non. Ces derniers, s'ils sont militaires, bénéficient, nonobstant le caractère massif des dommages infligés, de la présomption de respectabilité de leurs Etats, sous réserve toutefois que ceux-ci sortent vainqueurs du conflit dans lequel ils ont ordonné à leurs combattants de commettre des "actes d'Etat " objectivement criminels et donc punissables. A l'inverse, la tendance est répandue, notamment chez les vainqueurs du moment, de traiter par le droit commun de la délinquance crapuleuse et/ou terroriste, l'action d'authentiques maquisards, résistants, partisans et autres combattants travestis, d'autant plus que les moyens et procédés auxquels ils recourent sans être plus létaux que d'autres, sont parfois d'une rusticité qui renvoie aux formes anciennes de la barbarie. La contradiction est donc flagrante entre l'approche émotionnelle du combat suicidaire par la victime et la victimologie de guerre en général, qui finit toujours par l'absolution du vainqueur, quel que soit le type de procédé mis en œuvre.

Le recours à la métaphore juridique, quant à lui, conduit à s'interroger sur quelques aspects essentiels de la démarche suicidaire combattante. Elle a fait douter en premier lieu que le co-contractant du suicidant, au-delà de ses agents médiateurs ait une existence bien réelle. Passe encore pour le maussade agent d'un Etat bien bureaucratisé qui promet les primes d'après décès, mais qu'en est-il pour les Bons Dieux de tous les paradis, les allégories patriotiques, les sociétés idéalisées de demain? S'ils sont douteux, la réalité de leur engagement à remplir leur obligation de rémunération ne le sera pas moins. Ainsi se pourrait-il que le consentement du suicidant résulte d'un autoégarement ou des effets d'une propagande erronée ou dolosive. Pire, si ces entités érigées en interlocuteurs contractuels n'existaient pas ou s'il y avait erreur sur le contenu de leur obligation et/ou sur la moralité de celle-ci, c'est la validité même du contrat suicidaire qui s'en trouverait affectée. La mise en question de la moralité du suicide pour le sujet lui-même, et le débat spécifique lié à l'allégation de terrorisme montre, en outre, les fragilités d'une interprétation contractuelle du combat suicidaire.

$\mathrm{Au}$ demeurant, et en matière de conclusion, il serait possible d'avancer que l'acrobatie d'une interprétation contractuelle du combat suicidaire reste un exercice salutaire, propre à vérifier au passage la relative inefficacité stratégique d'un procédé présenté souvent comme imparable et, de ce fait, particulièrement redoutable.

Or, contractuel ou non, le suicide de combat n'intervient que dans des situations conflictuelles extrêmement critiques, tant sous sa forme prosaïquement escapiste ou déclamatoire sacrificielle que dans sa version meurtrière où le suicidant se tue pour tuer. Il souligne l'extrême faiblesse du parti qui y a recours et il n'y a pas d'exemple qu'il ait infléchi le cours d'une bataille, encore moins d'une guerre. Il n'est pas d'exemple non plus que sur la durée, le phénomène, à l'instar d'une épidémie, ne 
s'épuise et dépérisse, quitte à ressurgir ailleurs et sous d'autres formes au gré d'adaptations quasi virales à de nouveaux contextes.

L'emploi tactique de l'arme suicidaire relève de l'espérance du désespéré. Ses résultats à l'échelle de la conflictualité mondiale restent dérisoires et sont souvent contreproductifs. Les attentats du 11 septembre 2001 sont, à cet égard, exemplaires. Ils évoquent plus la survenance d'un "coup", certes sévère, mais hasardeux, que l'avènement d'une Troisième Guerre mondiale avec ce qu'un tel concept implique d'infrastructures, d'organisation, de coordination et de commandement. Leurs 3044 malheureuses victimes n'ont pas initié un processus qui aurait assuré le triomphe des « visionnaires » d'Al-Qaïda. Celui-ci n'a débouché que sur leur déconfiture et quelques dommages pour eux collatéraux. Ainsi, l'invasion de l'Irak ne les visait pas directement mais, en généralise le désordre, la fragmentation politique, le foisonnement de la violence et une banalisation des opérations-suicides promises de ce fait à un prochain épuisement, les guerres civiles qui s'ensuivent tendent à les chasser de l'actualité mondiale, eux et leur prophétie de contre-croisade planétaire mondiale.

Y aurait-il néanmoins un avenir significatif pour le suicide de combat en général et l'attentat-suicide en particulier? On serait tenté de répondre par la négative si on était bien sûr qu'aucun kamikaze de demain ne disposera d'un vrai procédé de destruction massive qui plongerait tout ou partie de la planète dans une Apocalypse, dont les commanditaires, politiquement suicidaires eux aussi et improbables survivants, auraient bien du mal à s'extirper.

\section{NOTES}

1. . Durkheim E., Le Suicide, Paris, PUF, coll. « Quadrige », 11e éd., (1re éd., 1897), 2002, p. 263.

2. . Flaubert à Louise Collet, cité par Minois G., Histoire du suicide, la société occidentale face à la mort volontaire, Paris, Fayard, 1995.

3. . « Du Premier ministre [sud-coréen] aux fermiers tous disent que "Lee Kyung-hae" est mort en martyr ", rapporte Libération, 23 septembre 2003. Nous devons la communication de cette citation à Aline Gérard, journaliste au Parisien.

4. Selon l'envoyé spécial du journal Hankyorek de Séoul, ibid.

5. . Durkheim E., op.cit., p. 20.

6. . Nous entendons par «terroristes" les actions violentes menées contre des objectifs non militaires par des non-militaires.

7. . Ce mot, qui reviendra à maintes reprises au cours de ce texte pour des raisons de style, n'est pas à prendre dans son sens technique religieux. Il ne signifie ici rien d'autre que le don spontané ou sollicité, total ou partiel, de soi.

8. Alphonse Baudin, député à l'Assemblée législative (1849) de la IIe République, tué le 3 décembre 1851, sur une des barricades élevée à Paris en protestation républicaine au lendemain du coup d'Etat du prince-président Louis Napoléon Bonaparte, préludant au rétablissement de l'Empire.

9. . Durkheim E., op.cit., p. 238. 
10. . Genres escapistes, agressifs, oblatifs, ludiques regroupant respectivement onze espèces : fuite-deuil-châtiment, crime-vengeance-chantage-appel, sacrifice-passage, ordalie-jeu. Voir Baechler J., Les Suicides, Paris, Calmann-Levy, 1975.

11. . Pinguet M., La Mort volontaire au Japon, Paris, Gallimard, 1984, p. 36.

12. . Durkheim E., op. cit., p. 240.

13. . Barrois C., Psychanalyse du guerrier, Paris, Hachette, coll. « Pluriel », 1993, p. 33.

14. . Extrême pauvreté et désespérance sociale seraient, selon Hassan Nour, l'une des causes déterminantes des attentats-suicides marocains: "Sociologie du combat suicidaire au Maroc (attentats du 16 mai 2003) ", inédit.

15. . Géré F., Les Volontaires de la mort, Paris, Bayard, 2003, p. 136 et suivantes.

16. Stavroguine dit : "Celui à qui il sera indifférent de vivre ou de ne pas vivre, celui-là sera l'homme nouveau. Celui qui vaincra la souffrance et la peur, celui-là sera lui-même dieu ", in Dostoievski F., Les Possédés, Paris, Gallimard, 1972, p. 105.

17. L'argument selon lequel la représentation du Paradis d'Allah promis à ses élus comme un lupanar offert aux ébats sans fin de soudards libidineux nous semble relever de l'islamophobie la plus indigente malgré le réalisme suggestif du texte coranique: "des Houris aux grands yeux, semblables aux perles seront la récompense de leurs actes, [d'autant plus] qu'elles resteront toujours vierges, aimantes et du même âge ", Coran, S.56, V.21-23, 35-36 (traduction de Henry Mercier).

18. . "L'honneur guerrier, synthèse des vertus militaires, a pour épreuve de vérité, la mort (donnée ou reçue) ", écrit Claude Barrois, op. cit., p. 199.

19. . Baechler J., op. cit., pp. 221-222.

20. . " "kamikaze tokubetsu kögekitai” (forces d'attaque spéciale du vent des dieux). 2198 pilotes se sacrifièrent. 34 navires américains furent coulés et endommagés ", Pinguet M., op. cit., p. 354.

21. . Ibid., p. 254

22. . Ibid., pp. 255-256.

23. . Ibid., p. 260.

24. . Ibid., pp. 258-259.

25. . Voir «Les entreprises para-privées de coercition : de nouveaux mercenaires ? ", Cultures \& Conflits, $n^{\circ} 52$, hiver 2003, Paris, l'Harmattan, 2003.

26. . Géré F., op. cit., p. 162.

27. . Pinguet M., op. cit., p. 259.

28. . Bozarslan H., « Temps, espoir et violence », inédit.

29. . Selon la définition du professeur Jacques Chestin cité par Raymond G., Droit civil, Paris, Editions Litec, 1996.

30. . Voir par exemple Raymond G., op. cit., pp. 197-229.

31. . Fadwa Touqan, poétesse palestinienne, citée par Laxalt T., " Naplouse, la reine assiégée ", Ethno $\mathrm{n}^{\circ} 1$, novembre 2004, p. 108.

32. . Le Coran est des plus explicites qui énonce tout crûment à l'intention des combattants : «Allah a acheté aux Croyants leurs personnes et leurs biens contre don à eux du Jardin. Ils combattent dans le chemin d'Allah. Ils tuent ou sont tués. Ils combattent dans le chemin d'Allah », Le Coran S.IX, v.111, trad. par Régis Blachère, Paris, Maisonneuve et Larose, 1980, p. 22. 33. . Pierre Legendre a écrit des pages fort éclairantes sur «l'encadrement nationaliste, où se trouve porté jusqu'au sublime l'amour du pouvoir » et bien d'autres choses encore sur « le culte fanatique des chefs", l'» utopie patriote ", l'» Etat sacré», le «Père omniscient », l'» idée canonique du pontife », la « possession du sujet par l'institution », etc. Voir L'Amour du censeur, Paris, Le Seuil, 1978, p. 187 et suivantes.

34. . C'est le type même du sacrifice christique offert à l'imitation des fidèles, pas seulement en ambiance chrétienne. Les sacrifiés volontaires islamiques "dans la voie de Dieu », ont bien conscience de contribuer à la sanctification de la communauté. Voir Seqqat H., Bayna al-istishâd wa al-irhâb [entre sacrifice et terrorisme], Fès (Maroc), Zaman, 2003, p. 10. 
35. . Raymond G., Droit civil, op. cit., p. 197.

36. . Ibid. p. 218.

37. . Sur la dépénalisation du suicide, voir Mullier A., Le Droit face à la mort volontaire, mémoire de DEA, université Lille-II, 2001-2002, (http://edoctorale74.univ-lille2.fr), assorti d'une excellente bibliographie. "Punir un mort ne sert à rien", note-t-il sobrement (p. 26). On remarquera cependant que la provocation au suicide, forme aggravée de l'incitation, fait en France l'objet d'une répression spécifique : voir l'article 223-13 et 14 du code pénal (loi du 31 décembre 1987, suite à la parution du livre de Guillon C., Le Bonnec Y, Suicide mode d'emploi, Alain Moreau, Paris, 1982). De manière plus lapidaire la sagesse arabe énonce la vanité des sanctions postmortem : « lâ lijrhin bimiyit .îlâm » (« aucune blessure ne fait souffrir un mort »).

38. . Voir notre article « Paix de compromis et tradition musulmane », Défense nationale, $n^{\circ} 8-9$, août-septembre 2000, p. 125. L'Eglise catholique, quant à elle, a condamné globalement le suicide au concile d'Arles (452) et de Prague (563), Durkheim E., op. cit., p. 370. Quant au judaïsme, « il a développé une casuistique complexe et une description nuancée du processus suicidaire » au terme desquels il y a lieu de distinguer entre le suicide de résistance, légitime, et ce qu'on pourrait appeler suicide de convenance blâmable ; voir Dufour Y.R., Le Suicide dans les textes de la tradition juive, www.modia.org. Les colons israéliens jusqu'au-boutistes n'hésitent pas, quant à eux, à prôner pour autant que de besoin un «sacrifice de soi » («mesirat nefesh » en hébreu) allant jusqu'au suicide (Barnavi E., « Désengagement », Le Monde 2, juin 2005, p. 32).

39. . Ndrl : Cette phrase a été attribuée à Pierre Jacques Etienne, Baron de Cambronne, le 18 juin 1815 lors de la bataille de Waterloo.

40. . Parmi lesquels François Géré épingle justement le nihilisme érigé par Netchaïev en religion, lequel nihilisme n'est pas sans points paradigmatiques communs avec le suicide terroriste contemporain, aux antipodes du mythe du « zéro mort ». Géré F., op. cit., p. 72.

41. . Epître saint Jean 316.

42. . " Harakiri », sur les suicides de remontrance et de « bonnes manières », voir Pinguet M., op. cit., pp. 236-237.

43. . Lapeyre P., sous-lieutenant au 5e régiment de tirailleurs sénégalais, «Commandant le poste de Beni-Derkoul [Maroc] [...], a tenu en échec, pendant soixante et un jours, un ennemi ardent et nombreux [...]. Le 14 juin 1925, submergé par le flot ennemi, a fait sauter son poste plutôt que de se rendre, ensevelissant à la fois sous les ruines le reste de sa garnison et ses assaillants ». On sera frappé par l'analogie, à trente siècles de distance, entre les conduites du jeune officier et du Samson des premiers temps d'Israël, sauf que Samson n'était pas militaire et que les Philistins n'étaient pas armés.

44. . Protocole additionnel aux Conventions de Genève de 1949, nº 1977, art. 37-1 et 48.

\section{RÉSUMÉS}

Une approche typologique de la mort polémique volontaire, militaire ou militante, meurtrière ou purement déclamatoire, révèle dans la durée une diversité de conduites individuelles aux antécédents complexes : de la compulsion pathologique au calcul stratégique. Puiser dans la métaphore du contrat, comme on dit dans le banditisme de droit commun, une grille d'analyse du suicide de combat présente un grand intérêt méthodologique, tant pour l'identification des auteurs et cibles de l'acte suicidaire, les causes de celui-ci, son objet et ses effets. Dépouillée de 
ses pollutions émotionnelles, l'appréhension du " kamikazat " révèle ainsi les limites d'une conduite plus désespérée qu'efficace.

The typological approach of voluntary polemical death, whether it is military or militant, murderous or purely declamatory, reveals a diversity of individual behaviours with complex antecedents, ranging from pathological compulsions to strategic calculation. To draw on the metaphor of the contract, as with common law crimes, in the analytical framework of combat suicide represents a great methodological advantage both in terms of the identification of the authors and the victims of the suicidal act and of its causes, object and effects. Once all emotional pollutions have been put aside, the apprehension of "kamikaze operations" reveals the limits of a conduct that is more desperate than efficient.

\section{INDEX}

Keywords : conflicts, violence, war

Mots-clés : conflits, kamikaze (missions suicide), sociologie des conflits (polémologie), violence

\section{AUTEUR}

\section{LOUIS-JEAN DUCLOS}

Après une carrière militaire qui l'a conduit à pratiquer un certain nombre de théâtres d'opérations extérieures, Louis-Jean Duclos a effectué une seconde carrière de chercheur en relations internationales, orientation Proche-Orient. Auteurs d'articles sur des sujets de géopolitique afférents à cette région. 